\title{
EXPECTATIVAS E PERCEPÇÕES DOS ESTUDANTES DO CURSO TÉCNICO EM ENFERMAGEM COM RELAÇÃO AO MERCADO DE TRABALHO*
}

\author{
Thiago Luis de Andrade Barbosaㄹ, Ludmila Mourão Xavier Gomes², Tatiana Carvalho Reis ${ }^{3}$, Maisa Tavares d \\ e Souza Leite
}

\begin{abstract}
${ }^{1}$ Mestrando em Ciências da Saúde pela Universidade Estadual de Montes Claros (Unimontes). Coordenador da Epidemiologia da Secretaria Municipal de Saúde de Januária. Minas Gerais, Brasil. E-mail: tl_andrade@yahoo.com.br

${ }^{2}$ Doutoranda em Ciências da Saúde pela Universidade Federal de Minas Gerais. Minas Gerais, Brasil. E-mail: ludyxavier@ yahoo.com.br

${ }^{3}$ Aluna do Curso de Graduação em Enfermagem da Unimontes. Bolsista de Iniciação Científica da Fundação de Amparo a Pesquisa de Minas Gerais (FAPEMIG). Minas Gerais, Brasil. E-mail: tatycnn@hotmail.com

${ }^{4}$ Doutora em Ciências. Docente do Departamento de Enfermagem da Unimontes. Professora Colaboradora do Mestrado em Ciências da Saúde da Unimontes. Minas Gerais, Brasil. E-mail: maisa.leite@unimontes.br
\end{abstract}

\begin{abstract}
RESUMO: Estudo com abordagem qualitativa e descritiva cujo objetivo foi identificar as percepções e expectativas dos estudantes do curso técnico em enfermagem com relação ao mercado de trabalho. A coleta de dados ocorreu em outubro de 2009, com a utilização da entrevista semiestruturada com 12 estudantes do curso técnico de enfermagem. Os dados foram submetidos à análise de conteúdo. As entrevistas evidenciaram importantes questões relacionadas à empregabilidade da enfermagem entre as categorias, às áreas de atuação profissional e à inserção do técnico na equipe de saúde. Os resultados mostraram a necessidade da continuidade da discussão sobre o tema, na busca de incrementar e melhorar a formação do técnico em enfermagem frente ao mercado de trabalho. Observou-se a existência de fatores negativos, como a discriminação e o pouco reconhecimento social. Conclui-se que o futuro egresso busca, além do emprego, a inserção na sociedade, para a sua valorização, enquanto profissional de saúde.
\end{abstract}

DESCRITORES: Estudantes. Mercado de trabalho. Enfermagem. Educação em Enfermagem. Educação técnica em enfermagem.

\section{EXPECTATIONS AND PERCEPTIONS FROM BRAZILIAN NURSING TECHNICIAN STUDENTS WITH RESPECT TO THE LABOR MARKET}

\begin{abstract}
The objective of this study with its qualitative and descriptive approach was to identify the perceptions and expectations nursing technician school students with respect to the labor market. The data was collected in October of 2009 using semi-structured interviews among 12 nursing technician students. The data was submitted to content analysis, which demonstrated that the interviews revealed important issues related to nursing employability among the categories, the areas of professional activity, and the integration of nursing technicians within the health care team. These results show the need for further discussion within the theme, seeking to increase and improve nursing technician formal education for the needs of the labor market. It beheld the existence of negative factors such as discrimination and limited social recognition. In conclusion, the future student seeks, beyond employment, his/her insertion in society for appreciation as a health care professional.
\end{abstract}

DESCRIPTORS: Students. Job market. Nursing. Education, nursing. Education, nursing, associate.

\section{LAS EXPECTATIVAS Y PERCEPCIONES DE LOS ESTUDIANTES DEL CURSO TÉCNICO DE ENFERMERÍA CON RELACIÓN AL MERCADO DE TRABAJO}

RESUMEN: Estudio cualitativo y descriptivo con el objetivo de identificar las percepciones y expectativas de los estudiantes del curso técnico de enfermería con relación al mercado de trabajo. La recolección de los datos se hizo en octubre de 2009, por medio de entrevistas semi-estructuradas con 12 estudiantes del curso técnico de enfermería. El examen de los datos se hizo por medio del Análisis de Contenido. Las entrevistas revelaron aspectos importantes sobre la ocupación de enfermería entre sus categorías, las áreas de desempeño profesional y la inserción del técnico en el equipo de salud. Los resultados muestran la necesidad de continuar el estudio sobre el tema para mejorar la formación del técnico de enfermería y su inserción en el mercado laboral. Se observó la existencia de factores negativos, como la discriminación y poco reconocimiento social. La conclusión del estudio es que el futuro egresado busca, además del empleo, la inserción en la sociedad para la su valorización como profesional de salud.

DESCRIPTORES: Estudiantes. Mercado de trabajo. Enfermería. Educación en enfermería. Graduación en auxiliar de enfermería.

* $3^{\circ}$ lugar Prêmio Ingrid Elsen.

Texto Contexto Enferm, Florianópolis, 2011; 20 (Esp): 45-51. 


\section{INTRODUÇÃO}

A saúde constitui um importante setor no mercado de trabalho do país. Como os demais serviços de consumo coletivo, a saúde está vinculada a um conjunto de determinantes políticos e econômicos que permeiam o mercado de trabalho em geral. O dinamismo e as características de seus empregos foram influenciados pelo crescimento do sistema produtor de serviços e pela reforma do setor, os quais provocaram mudanças significativas na estrutura ocupacional, no perfil quantitativo e qualitativo da força de trabalho em saúde. ${ }^{1}$

O novo paradigma de saúde exige profissionais capacitados que atendam às demandas da população, bem como dos serviços de saúde. Nesse sentido, as mudanças na área da enfermagem foram significativas. Para a capacitação dos profissionais e pelo grande quantitativo de atendentes sem formação foi instituído o Programa de Formação em Larga Escala de pessoal de Nível Médio e Elementar, em 1981. Esse programa teve o objetivo de transformar os atendentes de enfermagem em auxiliares de enfermagem. ${ }^{2}$

Atualmente, sabe-se que a Enfermagem é composta por três categorias profissionais, formada por enfermeiros, técnicos e auxiliares de enfermagem. O técnico de enfermagem exerce atividade de nível médio, esta envolve orientação e acompanhamento do trabalho de enfermagem no grau de auxiliar, e participação no planejamento e no cuidado de enfermagem.

Na última década, o Ministério da Saúde (MS) qualificou os trabalhadores da área de enfermagem, por meio de um Projeto de Profissionalização da Área de Enfermagem (PROFAE). Integra o projeto, o curso de complementação da qualificação profissional de auxiliar de enfermagem, para técnico em enfermagem, tendo em vista a melhoria da qualidade de serviços de saúde públicos e privados..$^{3-4} \mathrm{O}$ processo de formação proposto pelo PROFAE visa a atender às exigências do SUS e apresenta três grandes eixos: oferta de cursos de nível fundamental e médio, formação profissional no nível de qualificação profissional (auxiliar de enfermagem) e complementação de qualificação profissional (técnico de enfermagem). ${ }^{4}$

Destaca-se que o processo de capacitação e formação de profissionais na saúde e, particularmente, na enfermagem, se inicia por meio da escolha de uma profissão. Essa, por sua vez, se constitui em um ato reflexivo. Essa escolha tem sido definida pela ascendência histórica do indivíduo, isto é, ao optar por uma determinada profissão ele sofre influência das experiências que teve ao longo de sua vida, de fatores internos e externos, dos familiares e do mercado de trabalho no qual está inserido. ${ }^{5}$ Isso inclui, ainda, sua capacidade de lidar com frustrações e conflitos, e também seus valores éticos. A opção profissional é vista como um processo de crescimento, de exploração de potencialidades, de identificação e aceitação de si próprio, de harmonização ou integração dos motivos individuais e sociais, que acabam refletindo em um trabalho de qualidade.

Além disso, pode-se englobar no contexto da opção profissional a questão da satisfação, entendida como um conceito multifacetado que engloba aspectos pessoais, vocacionais e contextuais da realidade do trabalho. ${ }^{6}$ Por outro lado, a percepção do mercado de trabalho está associada ao grau de decisão de carreira e ao otimismo quanto à inserção no mercado de trabalho. ${ }^{7}$

Reconhecer a motivação dos estudantes que ingressam em um curso de enfermagem ou técnico de enfermagem é fundamental, pois para que eles consigam terminar o curso, é necessário que estejam preparados para superar as dificuldades que enfrentarão neste período. ${ }^{5}$

A educação profissional deve estar orientada aos problemas relevantes da sociedade, de modo que a seleção de conteúdos essenciais deve ser feita com base em critérios epidemiológicos e nas necessidades de saúde. ${ }^{8}$ É importante destacar que, por parte dos estudantes da área de enfermagem (enfermeiros ou técnicos de enfermagem), ainda existe uma grande confusão entre a profissão escolhida e as características do curso e do mercado. ${ }^{6}$

A conclusão do curso técnico de enfermagem e o momento de inserção no mercado de trabalho pressupõem períodos propícios para o surgimento de preocupações relativas à escolha, configurando-se, então, momentos cruciais para investigação científica. Ademais, observa-se, no Brasil, uma carência de estudos que identifiquem as expectativas e percepções dos estudantes do curso técnico de enfermagem com relação ao mercado de trabalho.

Dentro desse contexto, esse estudo teve por objetivo identificar a percepção e as expectativas dos alunos de uma escola do curso técnico de enfermagem em relação ao mercado de trabalho.

\section{METODOLOGIA}

Trata-se de um estudo de caráter descritivo, com abordagem qualitativa, realizado em uma escola do curso técnico em enfermagem, na 
cidade de Januária, Minas Gerais. Os sujeitos da pesquisa foram 12 estudantes do curso técnico em enfermagem.

A pesquisa qualitativa não é baseada no critério numérico, a fim de garantir sua representatividade, mas privilegia os sujeitos sociais que detém os atributos que o pesquisador deseja conhecer. ${ }^{9}$ Participaram da pesquisa os estudantes do curso técnico em enfermagem matriculados na referida instituição, nos turnos matutino ou noturno, e que aceitaram participar da pesquisa. O número de entrevistados foi definido a partir da saturação de dados, ou seja, quando nenhuma informação nova foi referida pelos participantes. As entrevistas foram realizadas no mês de outubro de 2009, na própria escola, e tiveram a duração aproximada de 20 a 45 minutos.

Os dados foram obtidos por meio de uma entrevista semiestruturada, estabelecida mediante roteiro constituído de perguntas abertas. As entrevistas foram previamente agendadas, definindo-se local e horário mais conveniente para os alunos e pesquisadores para a aplicação da entrevista semiestruturada, e realizadas mediante assinatura do Termo de Consentimento Livre e Esclarecido. As informações obtidas foram anotadas e gravadas e, posteriormente, transcritas pelos pesquisadores.

Em seguida, os dados coletados foram analisados em categorias ou núcleos de pensamento. Procedeu-se utilizando a técnica de análise de conteúdo, ${ }^{9-10}$ que se compõe de três grandes etapas: 1) a pré-análise; 2) a exploração do material; 3) o tratamento dos resultados e interpretação. Após a transcrição das entrevistas, realizou-se leitura repetida e exaustiva, permitindo realizar a ordenação do conjunto dos dados obtidos. Iniciou-se, assim, uma primeira classificação para apreender as estruturas relevantes, possibilitando o desvelamento das categorias empíricas confrontadas à luz da literatura atual.

Os aspectos éticos da pesquisa foram considerados de acordo com a Resolução n ${ }^{\circ} 196$, de 10/10/96, da Comissão Nacional de Ética em Pesquisa do Ministério da Saúde, que estipula normas éticas regulamentadoras de pesquisas envolvendo seres humanos. Para garantir o anonimato, os estudantes do curso técnico de Enfermagem foram identificados como E1, E2, sucessivamente.

A presente pesquisa foi autorizada pelo Comitê de Ética em Pesquisa da Universidade Estadual de Montes Claros (Unimontes), por meio do Parecer Consubstanciado n ${ }^{\circ}$ 1621/ 2009.

\section{RESULTADOS E DISCUSSÃO}

A partir da análise dos discursos dos entrevistados emergiram as seguintes categorias: O Mercado de Trabalho da Enfermagem e das outras áreas da saúde; mercado de trabalho para os cursos: graduação em enfermagem e técnico de enfermagem; A inserção do técnico de enfermagem no Sistema Único de Saúde (SUS) e o mercado de trabalho em enfermagem.

\section{O mercado de trabalho da enfermagem e das outras áreas da saúde}

A enfermagem é uma das poucas profissões na qual o mercado de trabalho continua em expansão. As diversas áreas de atuação de enfermagem ampliam as oportunidades de empregabilidade, podendo ser uma das principais motivações que fazem com que os cursos de enfermagem tenham uma alta demanda. ${ }^{3-11}$

As exigências e as consequentes mudanças no mercado de trabalho para enfermagem são atribuídas à própria dinâmica do setor saúde, que vem associada a uma rede de determinantes políticos e econômicos, como é evidenciado no discurso abaixo: ${ }^{12}$

[...] eu acho que na área da saúde a enfermagem tem carência de profissionais. O mercado de trabalho de outras áreas é muito concorrido. A pessoa que está na área da enfermagem terá mais facilidade para conseguir emprego (E4).

O mercado de trabalho exige do profissional de enfermagem conhecimento diversificado para superar a falta de experiência e enfrentar a competitividade. ${ }^{12}$

O mercado de trabalho para a enfermagem hoje está com um acúmulo de profissionais. Antes não havia muitos profissionais. Agora, as pessoas estão investindo na formação de novos profissionais. Para sobressair nesse campo tem que ser o melhor (E11).

Conforme as falas dos participantes relatadas, a questão da empregabilidade é tema de grandes discussões no âmbito da enfermagem, sendo, por vezes, contraditórias. Apesar da redução da mão de obra intensiva, o setor saúde conseguiu ter um crescimento expressivo nas últimas décadas, em plena era tecnológica. O mercado de trabalho em saúde passa de menos de quatrocentos mil empregos na década de 1970 para cerca de um milhão e meio em 1992 e, para mais de dois e meio milhões em 2005. Nesse período, foram gerados o equivalente a 1.127.986 novos postos de traba- 
lho, destacando entre eles a categoria de técnico/ auxiliar de enfermagem, que passou de duzentos e sessenta mil para quinhentos e sessenta mil no período de 1992 a $2005 .{ }^{13}$ Contudo, deve-se fazer menção ao fato de que durante muitos anos houve uma restrição da oferta de empregos para técnico de enfermagem, o que resultou na utilização de uma força de trabalho não qualificada e sem formação específica no mercado, colocando em risco a saúde da população. ${ }^{14}$

A família possui papel importante no processo de escolha da profissão, dada a sua enorme relevância na vida de qualquer indivíduo. Além disso, foi observado ainda que a opção profissional dos discentes está associada ao interesse/afinidade pela área da saúde. ${ }^{15}$ Esses aspectos estão evidenciados nos depoimentos abaixo:

[...] eu gosto de enfermagem, meu esposo é enfermeiro e eu aprendi a gostar muito da profissão com ele. Por isso eu acho a enfermagem melhor que as outras áreas (E4).

[...] eu, particularmente, acredito que a enfermagem é uma área muito importante em relação à medicina, à odontologia. Ainda é arte de cuidar (E10).

O mercado de trabalho para a enfermagem cresce a cada dia e diversifica-se cada vez mais. Essa ampliação dos campos de atuação deve-se ao reconhecimento do profissional de enfermagem e ao seu preparo para atuar em diversas áreas da saúde.

A enfermagem está bem mais ampla hoje. A cada dia aumenta a necessidade de cuidado que é o que nós mais fazemos. Estamos ali para cuidar, para dar um suporte. Prestar os primeiros cuidados mesmo (E7).

A enfermagem participa do setor saúde com o maior contingente de trabalhadores, representado por cerca de $50 \%$ da força de trabalho, sendo que desse total $22 \%$ são pertencentes à categoria de técnico de enfermagem. Nesse sentido, a participação da enfermagem é imprescindível na consecução das metas institucionais, a partir das reformulações propostas tanto no setor público como privado. ${ }^{16}$

Eu acho que o trabalho da enfermagem ficou discriminado em relação às outras áreas, porque você observa que a medicina é um trabalho muito valorizado; o retorno em dinheiro é bem maior (E3).

Entretanto, vale dizer que o prestígio social de uma profissão está relacionada à valorização de uma categoria profissional frente à sociedade. A enfermagem precisa ocupar espaços e ter o reconhecimento enquanto uma das profissões essenciais da saúde. Necessita de uma agenda política específica que expresse os vários aspec- tos constitutivos de uma profissão, isto é, o saber específico, o mercado de trabalho, a forma de organização, que apontem sobre a necessidade de cada categoria participar mais efetivamente das diferentes iniciativas que dizem respeito à saúde, no sentido de ampliar a visibilidade do saber e do fazer em enfermagem. ${ }^{17}$

\section{Mercado de trabalho para os cursos: gradua- ção em enfermagem e técnico de enfermagem}

A enfermagem é uma profissão composta por três categorias de níveis diferenciados: enfermeiro, técnico e auxiliar de enfermagem. Tais profissionais integram a equipe de trabalho da área da saúde como um todo e necessitam se relacionar com outros membros da equipe multiprofissional. ${ }^{11}$

Embora seja representativa em relação ao total de empregos em saúde no Brasil, a absorção de cada categoria de enfermagem apresenta significativas diferenças. Atualmente, a categoria encontra-se dividida nas seguintes proporções: $41 \%$ são auxiliares de enfermagem, $44 \%$, técnicos de enfermagem e $15 \%$ enfermeiros. ${ }^{18}$

Acho que para o técnico tem mais vagas. O pessoal comenta que aqui fora é bem mais fácil arrumar emprego como técnico do que como enfermeiro (E1).

Apesar de estar se investindo muito na área da saúde, não está tendo emprego suficiente para tanto enfermeiro. Está surgindo muito técnico e enfermeiro (E2).

O mercado de trabalho prefere profissionais que já tenham o conhecimento acabado e que venham com conhecimento aplicável a ser consumido no momento da sua produção, sem que se façam investimentos institucionais no processo de capacitação. Em geral, não se compromete com a educação permanente, prefere-se receber o conhecimento e repassá-lo adaptado para o fazer sem maiores reflexões, como é destacado abaixo: ${ }^{19}$

Hoje o superior está mais fácil de arrumar emprego. Como há muito enfermeiro, as pessoas não vão deixar de colocar o enfermeiro para colocar o técnico. As pessoas preferem contratar mais o enfermeiro que o técnico, porque tem uma formação melhor (E11).

Nessa perspectiva, observa-se, por um lado, a valorização do desenvolvimento adequado de procedimentos manuais, sendo essa habilidade considerada como característica do bom profissional. Por outro lado, ressalta-se que a diferença do enfermeiro para os profissionais de enfermagem de nível médio está no deter o conhecimento do por quê, como e para quê se realiza um procedimento. Ou seja, afirmam que é essa relação saber/ 
fazer que diferencia o enfermeiro e o torna responsável pela equipe de enfermagem. ${ }^{20}$

No Brasil, o mercado de trabalho em saúde possui características particulares, resultantes da dinâmica da reforma do setor que foi permeada pelos investimentos decorrentes da política pública setorial e pelo desenvolvimento do mercado de planos e seguros de saúde. ${ }^{21}$

Merece destaque o fato de que o mercado requer do profissional competências técnicas. ${ }^{19}$ Nos últimos anos, técnicos e auxiliares de enfermagem têm procurado cada vez mais a graduação em Enfermagem. ${ }^{5}$

O curso superior de enfermagem é melhor. $\hat{E}$ sempre um nível mais elevado. O técnico de enfermagem é bom. Não há equipe de saúde sem o técnico. Mas tenho certeza que os meus colegas que passaram por aqui queriam o curso superior de enfermagem. Acredito que ele [curso superior de enfermagem] pode ajudar muito mais (E4).

A diversidade apresentada para inserção no mercado de trabalho exige que o profissional valide a sua competência para a empregabilidade e corrija suas fragilidades de forma permanente, por meio da educação continuada. ${ }^{12}$

As áreas de atuação da enfermagem são inúmeras, no que pese as atividades de promoção à saúde - de educação em saúde e de atendimento ambulatorial e de domicílio, no tratamento de doentes e na reabilitação - tanto nos domicílios, quanto nos hospitais e clínicas. Estes espaços configuram-se como abrangentes o suficiente para oportunizar a colocação dos profissionais no mercado de trabalho. Esta gama de possibilidades é um dos principais atrativos para que os cursos de enfermagem tenham grande demanda. ${ }^{11}$

No Brasil, o mercado de trabalho para enfermagem apresenta elevada oferta de emprego, principalmente na área pública, fato esse constatado pelo número de concursos abertos no país.

Eu prefiro o Prontoatendimento. É o que eu mais gosto. Identifico-me mais com as pessoas que chegam precisando de mais atenção. Essa parte me chama muito a atenção (E10).

Gosto mais do Prontoatendimento, porque lá você aprende no dia-a-dia no que acontece lá. Eu prefiro o convívio com aquilo que acontece no dia-a-dia (E9).

$O$ vínculo entre a aprendizagem continuada e a vida profissional deve ser um compromisso, em busca da adequação às novas exigências do mercado de trabalho e para uma assistência com qualidade. $^{12}$
Nos ambientes de urgência, os profissionais estão sujeitos à tensão emocional constante, pois os problemas presentes no cotidiano lhes parecem maiores do que os recursos disponíveis para resolvê-los. No entanto, a satisfação em trabalhar na unidade de urgência decorre da sensação de dever cumprido por conseguir salvar vidas, sobrepondo-se às dificuldades por eles encontradas. ${ }^{22}$

\section{A inserção do técnico de enfermagem no SUS e o mercado de trabalho em enfermagem}

A Constituição Federal de 1988 atribui ao SUS a missão de ordenar a formação de recursos humanos para a área da saúde, conforme o art. 200, inciso III da Constituição Brasileira. ${ }^{23}$ Nessa perspectiva, o MS vem desenvolvendo e apoiando diversas ações no campo da formação e desenvolvimento dos profissionais de saúde.

Na formação de recursos humanos em saúde, a discussão envolve a preparação e a qualificação dos trabalhadores para a saúde coletiva, vislumbrando a consolidação da Estratégia Saúde da Família (ESF), o SUS e a garantia dos seus princípios fundamentais. ${ }^{24}$

O SUS aumentou o acesso da população e a área de abrangência, e consequentemente a empregabilidade, demonstrando que ainda há muito a avançar na consolidação do sistema, inclusive nas mudanças da estrutura curricular dos cursos da saúde. No entanto, constatou-se que o perfil dos profissionais formados não é adequado o suficiente para prepará-los para uma atuação na perspectiva da atenção integral à saúde e de práticas que contemplem ações de promoção, proteção, prevenção, atenção precoce, cura e reabilitação. ${ }^{17}$

O processo de formação profissional é permanente durante a vida produtiva do indivíduo e envolve todo o contexto social e as situações de saúde/doença da população com o perfil profissional requerido pelo mercado. Esse processo compreende as interfaces entre o aprendizado durante o curso de graduação e a busca do autodesenvolvimento como facilitador para inserção no mercado de trabalho. ${ }^{12}$

A área da saúde está formando muitos profissionais. Numa cidade pequena ainda está muito bom. Assim, na cidade grande eu acho que não é tão fácil assim. O mercado de trabalho está mais saturado (E5).

É consensual que os fatores ligados às perspectivas de emprego se constituem como uma das questões centrais que influenciam os profissionais de saúde na tomada de decisão em relação à migração. ${ }^{25}$ Esses fatores podem ser percebidos no discurso acima. 
As transformações significativas no mercado de trabalho vêm ocorrendo de forma vertiginosa, nos últimos anos, implicando no reconhecimento da realidade através da realização de pesquisas que monitorem o resultado desse processo. A reorganização do sistema produtivo/econômico capitalista, refletida nas configurações do sistema ocupacional, é verificada nas novas relações de trabalho, nos novos formatos, regimes e modalidades de contratação que têm sido adotadas no âmbito da gestão dos recursos humanos. Por conseguinte, esse fato representa uma das premissas na agenda atual de pesquisadores, governantes e gestores na definição de prioridades na produção de bens e serviços nas esferas federal, estadual e municipal. ${ }^{26}$

Na área hospitalar é muito disputado. Você tem que sobressair mesmo. Agora se você quiser trabalhar em outra área. Pode cuidar de um idoso, trabalhar em um asilo (E3).

Percebe-se na fala acima que a área hospitalar, por ser uma área de boa empregabilidade para enfermagem no SUS, é alvo de grande concorrência e afirmação profissional.

Com a implantação da ESF, surgem novos postos de trabalho e uma nova organização do trabalho. A ESF constitui na principal política re-orientadora do modelo de Atenção à Saúde no país. Dessa forma, a formação dos trabalhadores em saúde deve acompanhar a realidade do SUS. Atualmente, a Atenção Primária à Saúde constitui numa área de empregabilidade com um novo paradigma,${ }^{27}$ voltado para promoção e prevenção, contrário ao modelo médico-hegemônico curativista. Esse fato é constatado no depoimento:

[...] cuidar e tentar prevenir, conversar, falar. Auxiliar e conversar, fazer visita domiciliar no Programa Saúde da Família (E6).

Além disso, a garantia da integralidade da atenção, da humanização da assistência, da construção da interdisciplinaridade e do trabalho em equipe representa o novo paradigma doSUS adotado nas ESF. Assim, o trabalho destacado como promoção e prevenção da saúde com a assistência voltada ao cuidado e às visitas domiciliares constitui na quebra do modelo médico hegemônico e na adoção dos princípios do SUS. ${ }^{28}$

\section{CONSIDERAÇÕES FINAIS}

A educação profissional de nível técnico em enfermagem deve formar profissionais com competências e habilidades para melhor entender a prática da enfermagem e a dinâmica de sua inserção no mundo do trabalho. No entanto, ao se abordar o mercado de trabalho de enfermagem em relação a outras áreas de saúde, percebeu-se que fatores, como o baixo retorno financeiro e a falta de reconhecimento social, são considerados importantes fatores para a satisfação profissional. O futuro egresso busca além do emprego a inserção na sociedade para a sua valorização enquanto profissional de saúde.

Recomenda-se a realização de novos estudos sobre a temática em outras regiões do país, considerando os aspectos culturais, novos cenários e atores sociais, ampliando conhecimento acerca do fenômeno.

Este estudo contribui para a produção de conhecimentos na área de Enfermagem com subsídios para novas pesquisas, no intuito de explorar melhor a realidade do mercado de trabalho para o técnico de enfermagem. Além disso, contribui para propor ações com vistas ao processo de formação de um profissional que esteja preparado para se inserir em um mercado que se encontra cada vez mais competitivo.

\section{REFERÊNCIAS}

1. Vieira ALS, Amâncio Filho A, Oliveira ES. Mercado de trabalho em saúde na região sudeste-Brasil: a inserção da equipe de enfermagem. Rev Latino-am Enfermagem. 2004 Jan-Fev; 12(1):134-8.

2. Bassinello GAH, Bagnato MHS. Os primórdios do Projeto Larga Escala: tempo de rememorar. Rev Bras Enferm. 2009 Jul-Ago; 62(4):620-6.

3. Costa CCC, Bezerra FJG, Machado MMT, Machado MFAS, Jorge AC, Furtado AAA, et al. Curso técnico de enfermagem do PROFAE-Ceará: a voz dos supervisores. Texto Contexto Enferm [online]. 2008 Out-Dez [acesso 2010 Jun 03]; 17(4):705-13. Disponível em: http://www.scielo. br / scielo.php?script=sci_arttext\&pid=S010407072008000400011\&lng=pt.

4. Ministério daSaúde(BR).PROFAE: profissionalização dos trabalhadores da área de enfermagem. Brasília (DF): MS; 2006.

5. Medina NVJ, Takahashi RT. A busca da graduação em enfermagem como opção dos técnicos e auxiliares de enfermagem. Rev Esc Enferm USP [online]. 2003 Dez. [acesso 2010 Jun 03]; 37(4): 101-8. Disponível em: http://www.scielo.br/scielo.php?script=sci arttext\&pid=S0080-62342003000400012\&lng=en.

6. Bardagi M, Lassance MCP, Paradiso ÂC, Menezes IA. Escolha profissional no mercado de trabalho: percepções dos estudantes formados. Psicologia Esc Educ. 2006 Jun; 10(1): 69-82.

7. Teixeira MAP, Gomes WB. Estou me formando...e agora? Reflexões e perspectivas de jovens formandos universitários. Rev Bras Orientac Prof. 2004 Jun; 5(1):47-62. 
8. Feuerwerker LCM. Educação dos profissionais de saúde hoje - problemas, desafios, perspectivas e as propostas do Ministério da Saúde. Rev Abeno. 2003 Jan-Dez; 3(1):24-7.

9. Minayo MCS. O desafio do conhecimento: pesquisa qualitativa em saúde. $10^{a}$ ed. São Paulo: HucitecAbrasco; 2007.

10. Bardin L. Análise do conteúdo. $4^{\mathrm{a}}$ ed. Lisboa (PT): Edições 70; 2006.

11. Ferreira MA, Oliveira BGRB, Porto IS, Anhorn CG, Castro JBA. O significado do Profae segundo os alunos: contribuição para a construção de uma política pública de formação profissional em saúde. Texto Contexto Enferm. 2007 Jul-Set; 16(3):445-52.

12. Carrijo CIS, Bezerra ALQ, Munari DB, Medeiros M. A empregabilidade de egressos de um curso de graduação em Enfermagem. Rev Enferm UERJ. 2007 Jul-Set; 15(3):356-63.

13. Machado MH, Oliveira ES, Moyses NMN. Tendências do mercado de trabalho em saúde no Brasil [online]. In: Conferência Internacional sobre Pesquisas em Recursos Humanos em Saúde. Rio de Janeiro, Brasil. 2010 Jun 10 [acesso 2001 Abr. 05]. Colegiado dos Secretários Municipais de Saúde do Estado de Minas Gerais (MG). Disponível em: http://www.cosemsmg.org.br/cosems/images/ fbfiles/files/artigo_tendencias_EM_REVISO.pdf.

14. Oliveira BGRB, Porto IS, Ferreira MA, Castro JBA. Perfil dos alunos ingressos nos cursos de auxiliar e técnico de enfermagem do Projeto de Profissionalização dos Trabalhadores da Área de Enfermagem (PROFAE) no Rio de Janeiro - Brasil. Rev Latino-am Enfermagem. [online]. 2007 Fev. [acesso 2001 abr. 05]; 15(1):127-33. Disponível em: http:/ / www.scielo.br/scielo.php?script=sci arttext\&pid=S0104-11692007000100019\&lng=en.

15. Spíndola T, Martins ERC, Francisco MTRF. Enfermagem como opção: perfil de graduandos de duas instituições de ensino. Rev Bras Enferm. 2008 Mar-Abr; 61(2):164-9.

16. Fundação Instituto Brasileiro de Geografia e Estatística. Pesquisa de Assistência MédicoSanitária. [CD-ROM]. Rio de Janeiro: IBGE; 2005.

17. Erdmann AL, Fernandes JV, Melo C, Carvalho BR, Menezes Q, Freitas R, et al. A visibilidade da profissão de enfermeiro: reconhecendo conquistas e lacunas. Rev Bras Enferm. 2009 Jul-Ago; 62(4):637-43.

18. Conselho Federal de Enfermagem - COFEN (BR). Distribuição dos Profissionais de Enfermagem. 2010 [acesso 201016 Jan]. Disponível em: http:/ /www. portalcofen.gov.br/Site/2007/default.asp
19. Peres AM, Ciampone MHT, Wolff LDG. Competências gerenciais do enfermeiro nas perspectivas de um curso de graduação de enfermagem e do mercado de trabalho. Trab Educ Saúde. 2008 Nov; 5(3):453-72.

20. Gomes AMT, Oliveira DC. Formação profissional e mercado de trabalho: um olhar a partir das representações sociais de enfermeiros. Rev Enferm UERJ [online]. $2004 \mathrm{Dez}$ [acesso 2010 Jan 25]; 12(3):265-71. Disponível em: http://www.revenf. bvs.br/scielo.php?script=sci_arttext\&pid=S010435 522004000300003\&lng=pt\&nrm=iso.

21. Barbosa NB. Regulação do trabalho no contexto das novas relações público versus privado na saúde. Ciênc Saúde Coletiva. 2010 Ago; 15(5):2497-506.

22. Salomé GM, Martins MFMS, Espósito VHC. Sentimentos vivenciados pelos profissionais de enfermagem que atuam em unidade de emergência. Rev Bras Enferm [online]. 2009 Nov-Dez [acesso 2010 Jun 01]; 62(6):856-62. Disponível em: http:/ / www. scielo.br/scielo.php?script=sci_arttext\&pid=S003471672009000600009\&lng=pt.

23. Brasil. Constituição da República Federativa do Brasil. Brasília: Diário Oficial da República Federativa do Brasil, 05 out 1988 [acesso 2010 Mai 12]. Disponível em: http://www.planalto.gov.br/ ccivil_03/Constituicao/Constituiçao.htm.

24. Marsiglia RMG. Instituições de ensino e o Programa Saúde da Família: o que mudou? Rev Bras Saúde Família. 2004 Mar-Abr; 5(7):30-41.

25. Varella TC, Pierantoni CR. A migração de enfermeiros: um problema de saúde pública. Rev Bras Saude Matern Infant [online]. 2007 Abr-Jun [acesso 2010 Jun 01]; 7(2):199-211. Disponível em: http:/ / www.scielo.br/scielo.php?script=sci arttext\&pid=S1519-38292007000200011\&lng=en.

26. Bezerra O. Dinâmica e características do mercado de trabalho do setor saúde. In: Castro JL, organizadora. Gestão do trabalho no SUS: entre o visivel e o oculto. Natal (RN): Editora Observatório RH NESC/UFRN; 2007. p. 201-16.

27. Villa EA, Aranha AVS. A formação dos profissionais da saúde e a pedagogia inscrita no trabalho do Programa de Saúde da Família. Texto Contexto Enferm [online]. 2009 Out-Dez [acesso 2010 Jun 01]; 18(4):680-7. Disponível em: http://www. scielo.br/scielo.php?script=sci_arttext\&pid=S010407072009000400009\&lng=en.

28. Costa RKS, Miranda FAN. Sistema Único de Saúde e da família na formação acadêmica do enfermeiro. Rev Bras Enferm. 2009 Mar-Abr; 62(2):300-4. 